\title{
Computer-Assisted Cognitive Re-Training as An Intervention for Children with Specific Learning Disability: A Review
}

\author{
Gargi Bansal ${ }^{1 *}$, Anand Pratap Singh ${ }^{2}$
}

\section{ABSTRACT}

The review inspects the empirical literature on the efficacy of computer-assisted cognitive retraining of children with Specific learning disabilities (SLD). SLD children are characterized by an average and above-average IQ but there exist significant deficits in their language processing skills. Cognitive re-training is a training process that serves as remediation for people with underdeveloped cognitive abilities through intensive practice. It utilizes the principle of "brain plasticity" and is an endeavor to strengthen the deficit cognitive abilities of people by practicing various well-defined tasks and exercises. Cognitive re-training can be provided in both ways manualized or computerized. Computer-assisted re-training seems more interesting, innovative, is multisensory and motivating for children. This research review aims to put together the primary research done in the area and tries to evaluate the effectiveness of using such intervention on children with a specific learning disability. In a country like India which has a vast \& widespread population reaching out to children with a specific learning disability by using manualized intervention seems a distant reality, together with a handful of trained therapists working in the field. Keeping in view such circumstances there is an urgent need to identify ways which can be used as an intervention for the mass population and in remote areas of the country. In doing so, this review also attempts to lay a base and explore the possibility of utilizing this novel way of providing interventions to children with Specific learning disability.

\section{Keywords: Brain Plasticity, Manualized, Average IQ, Language Processing Skills, Multisensory}

$\mathrm{I}$ ndividuals with Disabilities Education Act (IDEA,2018) defined Learning Disability as "A disorder in one or more of the basic psychological processes involved in understanding or in using spoken or written language, which may manifest itself in an imperfect ability to listen, think, speak, read, write, spell or to do mathematical calculations."

According to Diagnostic and statistical manual of mental disorders (5th edition, DSM 5: APA 2013) Learning disability category is renamed as "Specific learning disability". it is characterized by the presence of persistent difficulties in reading, writing, arithmetic, or

\footnotetext{
${ }^{1}$ Research Scholar department of psychology and mental health Gautam Buddha University, India

${ }^{2}$ Assistant Professor and Head of the department of psychology and mental health Gautam Buddha University, India *Corresponding Author
}

Received: September 22, 2021; Revision Received: November 10, 2021; Accepted: November 27, 2021

(C) 2021, Bansal G. \& Singh A. P.; licensee IJIP. This is an Open Access Research distributed under the terms of the Creative Commons Attribution License (www.creativecommons.org/licenses/by/2.0), which permits unrestricted use, distribution, and reproduction in any Medium, provided the original work is properly cited. 


\section{Computer-Assisted Cognitive Re-Training as An Intervention for Children with Specific Learning Disability: A Review}

mathematical reasoning skills during formal years of schooling among children, despite getting conventional instructions and socio-cultural opportunities. Such children exhibit underachievement in various academic areas notwithstanding average or above-average intelligence. These individual's difficulties must not be better explained by developmental, neurological, sensory (vision or hearing), or motor disorders (DSM 5, 2013)

Specific learning disability is of 3 major types i.e., Dyslexia (a disorder of reading and comprehension), Dysgraphia (a disorder of writing) \& Dyscalculia (i.e., a disorder of mathematical calculations). The major one affecting almost $80 \%$ of the total children identified with a learning disability is dyslexia (Shaywitz., 1998). Dyslexia is the root cause of most learning impairments.

\section{Neurobiology of Learning Disability}

Learning disability is described as a neurobiological disorder as people with a learning disability have brains that learn differently because of differences in brain structure/brain functions (Golden, 1982). The 3 important areas of the brain responsible for reading take place are in the left hemisphere of the human brain. These are the Broca's area, Parieto temporal area (involved in word analysis), and Occipito-temporal area (responsible for reading fluency) (Shaywitz S., 2004). Several studies using neuro-imaging technologies (PET, $\mathrm{f}(\mathrm{MRI})$ ) have indicated that the left hemisphere reading system is dysfunctional in people with learning \& reading difficulties (Brunswick, 1999). Though struggling readers try to compensate for the deficits in their reading system through developing alternate reading system in the right hemisphere but unfortunately, they are unable to develop the highly efficient word-form region, and thus they can read, but not as efficiently and fluently as their similar-aged counterparts (Shaywitz S., 2003).

It has also been established through various fMRI studies that, learning disabled people have significant deficits in working memory, metacognitive skills, and self-regulation i.e., in their higher-order cognitive functioning which is also called executive functioning skills (K.H.Pribram, 1973). These are the functions of the prefrontal cortex.

Apart from these specialized brain regions, both the visual and the auditory systems play a crucial role in enabling humans to read and write. If there exists a problem in either of the two, then developing precise reading and comprehension skills is difficult and it also creates complexities in other verbal processing tasks. It has been found in studies by (Schulte-Körne G1, 1998) that dyslexics have a specific speech processing deficit at the sensory level which can serve as early identification of children at risk at an early age.

\section{Neuroplasticity \& Cognitive re-training in Learning disability}

Recent researches show that the brain can improve its deficit functioning if exposed to a proper training environment (Kolb, 2011), this adaptive ability of the brain which makes it highly responsive to environmental influence is known as "Brain-plasticity" or "Neuroplasticity" (DeFelipe.J, 2006) Several pieces of research provide evidence that plasticity is a function of an adult brain (Maguire EA, et al., 2000.) but it may occur during childhood only (Neville H, 2002). Increased functional connectivity and specialization of cortical circuits are the important features of a developing brain during childhood, thus it is unsurprising that the human brain is highly "adaptive" at this time, and by providing appropriate training its structure, function, and connections can be re-established. "Cognitive re-training" is one of the most effective methods of enhancing brain functions as it follows 


\section{Computer-Assisted Cognitive Re-Training as An Intervention for Children with Specific Learning \\ Disability: A Review}

the principle of providing repeated practice within a component cognitive skill which in turn shows positive results in both behavioral efficiency and cognitive functioning of an individual. Cognitive re-training \{rehabilitation\} can be defined as" a complex collection of techniques designed to enhance perception, attention, comprehension, learning, remembering, problem-solving, reasoning, and so forth (Callahan, 2001), (Cavanaugh, 1985) (Evans J., 1996); (Patten, 1990) in individuals who have impairments in the above said areas".

In simpler words, Cognitive re-training is an art and science of restoring the underdeveloped mental processes and teaching compensatory strategies to individuals who are intellectually disabled or have a minimal brain injury. The role of cognitive re-training in strengthening the deficient mental faculties is like the role of cardiovascular exercises in strengthening the muscles of our body. "Cognitive Re-training" is one of the most efficient and established ways of providing interventions in learning disabilities. This paper will majorly focus on cognitive re-training as an effective way of intervention for learning disabled children.

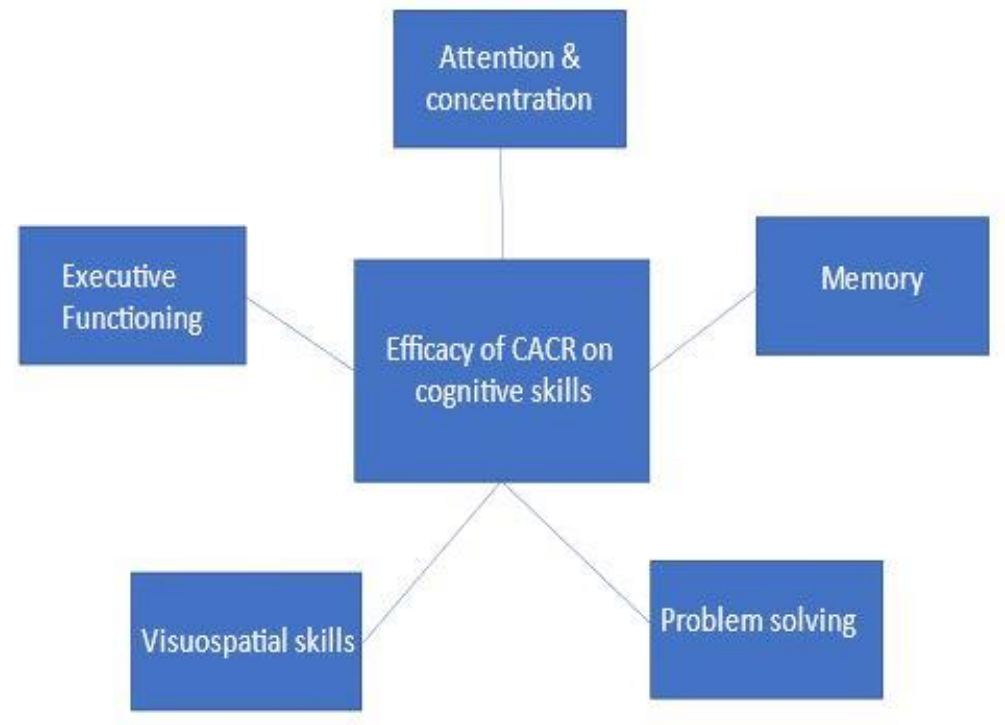

\section{Fig 1 shows the cognitive skills which can be enhanced using cognitive re-training approach of intervention}

There are 2 major ways of providing cognitive re-training to learning disabled individuals.

1. Manualized cognitive re-training: Manualised way of providing cognitive re-training involves the individualized guided practice on a set of standardized tasks designed by a specialist to reflect cognitive functions, such as attention, problem-solving, memory, or any other cognitive deficits which the client is facing. These tasks are presented to the client in the form of paper-pencil tasks. These tasks can either be tailormade as per individual requirements by the therapist or the therapist can decide to use standardized tasks available. Various studies are done in which the effectiveness was proved of using manualized cognitive re-training among children with a specific learning disability. (Rozario.J., 1994); (Hayward, 2007); (Das J. P., 1995); (Brown, 1996); (Das J. P., 2000).

In India, several studies were done using manualized cognitive re-training to provide remediation to children with a learning disability. (Malhotra S. R., 2009) concluded in their 


\section{Computer-Assisted Cognitive Re-Training as An Intervention for Children with Specific Learning Disability: A Review}

research that manualized cognitive re-training over 36 hours can help to partially remediate cognitive deficits in children with a learning disability and improve their scholastic performance. Another important piece of research done by (Malhotra S. \&., 2010) compared the efficacy of both cognitive re-training and remedial education as an intervention to children with a learning disability. The authors finally concluded that the best way to treat learning disabilities among children is to club both these methods of remediation and use a blended approach.

A manualized way of providing cognitive retraining is a traditional way of providing remediation by a therapist. Now let us discuss the uniqueness of computer-assisted cognitive re-training.

2. Computer-assisted cognitive re-training - Computer-assisted cognitive re-training provides cognitive remediation to clients in need of it through the medium of computers. The cognitive re-training this way includes various tasks and activities which are in the forms of games or any other interesting format designed by the experts. As it is a wellknown fact that Learning with the ample use of technology makes it more comprehensive and easier to understand for the clients. Using a virtual platform for remediation or intervention, furthermore, helps both the clients and educators as, it enables clients to take charge of their learning (Vincent, 1992). The audio-visual approach keeps the client involved through its interactive properties. Simultaneously, it gives a notion of control over their learning process as the client can complete sessions at their own pace (Pantelidis, 1993), making mistakes until they gain mastery over their concepts (Salem-Darrow M, 1996).

Computer-assisted cognitive retraining seems to be interesting for the client and its multisensory nature helps to target and stimulate various sense receptors like auditory and visual systems simultaneously. It is time-bound, can be administered remotely, costeffective, process-oriented, and adaptive which makes it more desirable to use.

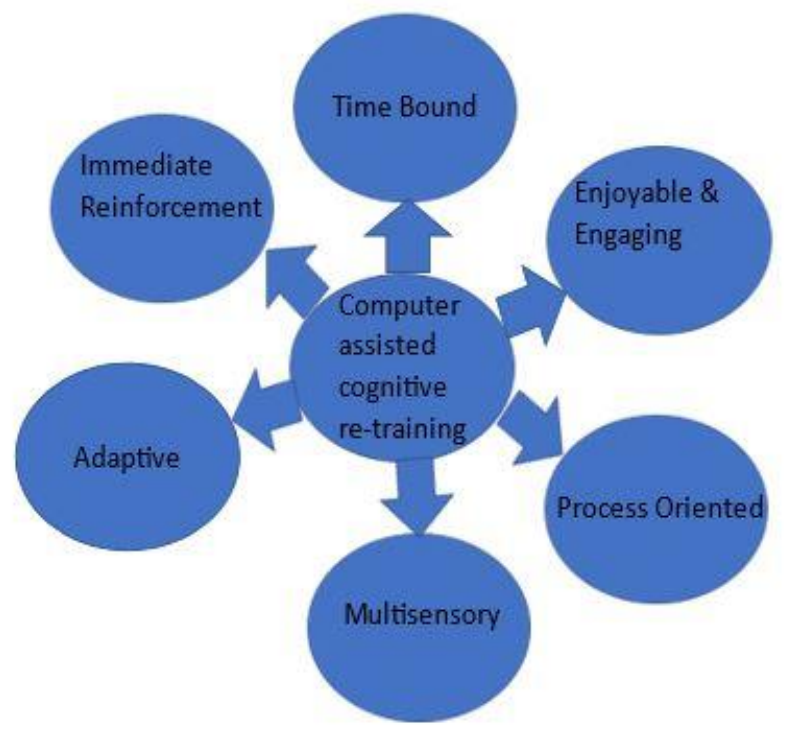

Fig 2 Benefits of using computer assisted intervention over manualized mode of intervention 


\section{Computer-Assisted Cognitive Re-Training as An Intervention for Children with Specific Learning Disability: A Review}

This paper focuses on how past research has proved the efficacy of computer-assisted cognitive retraining in improving various cognitive functions in children with a learning disability. It aimed to identify: -

1. The efficacy of computer-assisted cognitive re-training as an intervention for children with learning disabilities, the use of computer-assisted remediations to improve various deficit skills of children with learning disabilities like comprehension, auditory \& visual perception, working memory \&executive functions, decoding skills, etc.

2. It also aims to showcase the studies done in India using computer-assisted interventions on children with a learning disability, the gaps, and the need for future studies to be done

3. Finally, the paper presents how the use of computer-assisted interventions can serve as a major scaffolding in the remediation of specific learning disabilities among children at a mass level.

Computer-assisted cognitive re-training to improvelenhance:

1) Reading and comprehension skills

It has been observed that the phonologically mediated reading intervention would scaffold in building their fast-paced word form system and thus plays a very important role in improving the reading skills of struggling readers. (Mioduser, 2000) demonstrated the efficacy of computer-assisted reading intervention among children with learning disabilities. In the above study, computer-assisted reading interventions were compared to just printbased interventions and standard special education programs with no specific training for reading. The outcome of this study proved that children who were given computer-assisted remediations, such children had made a remarkable development in their phonological awareness, word recognition, and letter naming skills juxtaposing to the other group of children who received just print remedial materials (without the computer) and the standard special education program with no specific reading training.

(Rogowsky, 2013) evaluated the efficacy of computer-assisted cognitive and language training on the reading and writing skills of college students. The study also postulates the role of the computer-based learning program in building up basic auditory processing, spoken language skills, cognitive and reading skills in college-going students. The results of this study added value to already existing literature regarding the effectiveness of using a structured, advanced, perceptual/cognitive, language, and reading intervention program for struggling college-going students who are past the primary and secondary school level.

\section{2) Auditory and visual perception}

Both the visual and the auditory system plays a crucial role in enabling humans to read. An important study by (Pollatsek, 1982) specifically points out the importance of eye movements during reading by humans. The deficits in the Magnocellular pathway which is responsible for the processing of information about the position and movement of stimuli may affect the reading abilities of an individual.

Another important claim that dyslexic children have difficulty processing rapidly changing or brief auditory inputs came from a study by (Tallal P. M., 1974). (Tallal P., Sept 30, 2015) mentioned that the underlying cause of untreated dyslexia is the focus of interventions that usually targets upgrading the reading skills of children, but reading difficulties are just a symptom whereas the cause is underlying auditory processing deficits. 


\section{Computer-Assisted Cognitive Re-Training as An Intervention for Children with Specific Learning Disability: A Review}

(Kujala T, 2001) in their study outlined the finding that even computer-assisted nonlinguistic, audio-visual training can produce neural changes and reading improvements among reading-impaired children. Such training when given to children who had reading difficulties resulted in plastic changes in the auditory cortex, marked by increased electrophysiological ill-matched negativity and quicker reaction times to sound changes. Importantly, such changes also resulted in improved reading skills among students.

\section{3) Decoding skills}

One of the major causes of reading disability is a challenge in decoding a written text which reduces the speed and accuracy of reading comprehension as it can be cognitively taxing and leaving fewer resources available for comprehension (Smythe, 2005).

(Macaruso, 2009) in their study showed how computer-assisted interventions can be used to improve decoding skills in struggling readers. Along with the improvement observed in decoding skills, another major finding of this study was that the students who scored the lowest in various reading tests seemed to benefit the most from computer-assisted interventions. Not just this, the positive gains were observed in the reading comprehension skills of children in the treatment group. As decoding skills often scaffold in a reader's comprehension abilities thus it can be concluded that improvement seen in reading comprehension among treatment group of students is the output of improved decoding skills.

\section{4) Working memory and Executive function}

There are several studies (Swanson, 2011) that very well establish the fact that working memory plays an important role in developing the reading skills of an individual. The underdeveloped capacity of the working memory leads to the development of various disorders in children related to learning and reading abilities, motor abilities, and language impairments (Gathercole, 2008). In a study by (Callaghan, 2016) Randomised controlled trial design was used to assess the effect of computer-assisted literacy intervention for young children to enhance their phonological skills. The results suggested that such interactive interventions can be quite useful in the upliftment of phonological skills, majorly if these learning difficulties are not connected to phonological working memory deficits.

There are several studies to show that the gains made by the children in their cognitive skills by using computer-assisted interventions remain intact for a long time. A major longitudinal study was done by (Gustafson, 2011) to ascertain the benefits of computer-based interventions targeting the reading skills of children with dyslexia. The study examines the effects of three computerized interventions on the reading skills of primary school-going children with dyslexia. This longitudinal intervention study included 130 subjects, out of which 30 served as normal controls whereas 100 children were assigned randomly to 3 intervention groups. Five test sessions were done throughout 1 year ( 2 before the intervention and 3 after intervention). Though improvement in reading skills was found among all the 3 groups of children. But the group that received the combined intervention of both ie decoding skill training and sentence level training showed greater improvement than the one with ordinary activities involving reading and writing. Another major beneficial outcome of the computer-based intervention was indicated by the longitudinal results which suggested that the group of students who received the combined training (computer-assisted decoding skills \&sentence level), no longer required any remedial education even when assessed after one year of the intervention. 


\section{Computer-Assisted Cognitive Re-Training as An Intervention for Children with Specific Learning Disability: A Review}

Another important research was done by (Law, 2017) to evaluate the effectiveness of computer-assisted intervention targeting phonological processing skills for normal kindergarten children in Hong Kong who learn English as a second language. Children in the experimental group and control group were taught with computer-assisted and penciland-paper teaching approaches, respectively. On doing post intervention analyses, it was revealed that the computer-assisted intervention had a statistically significant effect on improving the students' alliteration, phoneme segmentation, and rhyme skills compared to the control group. Furthermore, the effectiveness of significantly improving students' alliteration and rhyme skills with computer-assisted intervention remained sustained 10 weeks after the intervention.

(Seidman LJ, 2001) in their research showed that children with a learning disability have weak executive functioning skills. Executive functioning skills are the mental processes that empower us in planning, focussing attention, remembering instructions, and juggling between multiple tasks successfully. These skills play a crucial role in enhancing the learning and scholastic abilities of children. (Bracy, 1999) demonstrated the utility of computer-assisted cognitive skills training for improving the intellectual functioning of 12 to 14-year-old children. The findings of this study very well support the fact that computerassisted cognitive rehabilitation provided to middle school students has led to improvements in their IQ scores and improved academic performance stemming from an enhanced skill base which was superior to gains from rather just educational based interventions.

\section{Indian Studies}

Though not many but few studies are done in the Indian context to predict the effectiveness of computer-assisted intervention in learning disability. (Nisha, 2013) did a study to assess the efficacy of computer-assisted cognitive training in the remediation of specific learning disorders. This study employed a comparison between groups before and after, in control experimental design. 10 children with learning disorders between the ages of 8 and 15 years were sequentially assigned to either EBRT (education-based computer training) + CACT (computer-assisted cognitive training), or only EBRT. The results indicated that skills in attention, reading, reading comprehension, spelling, and arithmetic improved better in groups given computer-assisted cognitive training along with education-based remedial training than in groups given only education-based remedial training. However, the differences between group scores were not significant.

Another study was conducted by (Kumar, 2014) to determine the effectiveness of the computer-assisted instructional package (Games/ Simulations) developed by the researchers as Remedial teaching for learning disabled children. The experimental group students were exposed to a computer-assisted instruction package, while control group students were taught using the traditional teaching method having the same content used for the experimental group. The findings for this study stated that there was a significant difference between the mean score of fifth-grade students taught with computer-assisted instruction and those taught with the traditional method of teaching. These findings supported the fact computer-assisted instruction is highly effective in the remediation of learning disability and secondly computer-assisted instruction method was better than the traditional method in remediation of learning disability. 


\section{DISCUSSION}

After reviewing the research done in the zone of the effectiveness of computer-assisted cognitive interventions for learning disabled children, it is a well-established fact that computer-assisted intervention has an extra cookie point over the conventional mode of intervention. The judicial usage of these learning programs has shown a significant improvement in the visuospatial skills, executive functioning skills, phonemic awareness, and decoding skills of an individual as most of its training is provided in the form of audiovisual computer games (Bracy, 1999). Most such researches are done in the western context, but on evaluating the researches done in the Indian context it was found that there is a lack of studies done in this area and the researches which are done do not mention the characteristics and the target skills of the software used as an intervention. There is no clarity towards the nature of the software used as an intervention i.e., is the training material only education-based, or it works on training various cognitive abilities of children. However, it is a known fact that if the software used has only education-based training then the improvement which is desirable in a child's executive functioning and working memory cannot be attained. Additionally, these interventions were relatively short-term and do not clarify their long-term impact.

In a country like India which has a mostly Bilingual population, the pressure to learn 2 scripts \{i.e. Hindi or any other native language with English\} starts at a very early age for children. Learning two scripts becomes quite challenging for children who already have cognitive and learning deficits. If there are researches to show the efficacy of computerassisted interventions in enhancing the cognitive and reading skills of an individual, then it can certainly be called "Metamorphosis" for such individuals who face lifelong problems of learning disability. India, being one of the most populous countries on the globe, the extent of learning problems among school-going children are humongous (around 10\% of schoolgoing children suffer from SLD, (Kuriyan, 2018) and reaching to an effective intervention strategy which can be provided to many pupils simultaneously has become an exigency. Though there are numerous software's which claims their efficacy for providing interventions in learning disability (like braintrain.com, cogmed.com, scilearn.com, ReadOn.ai), but due to lack of research in this area, none has proved its efficacy for remediating specific learning disabilities among Indian children. Hence there is a need to conduct research that aims to identify an effective software that can be used with Indian children.

Another important point that brings in to focus the urgent need of doing research using computer-assisted intervention in the Indian context is that the ASER (Annual State education Report, 2018) found that just $50 \%$ of grade 5 children in rural India could read a class 2-level text, which is quite a disturbing fact for a country which has Right to Education (RTE) in place since last 1 decade. RTE can only be made a success if India steps forward towards inclusive education and to attain that goal computer-assisted learning intervention can prove to be an important tool in the armory of educators. Lastly, if the effectiveness is proved for such computer-assisted cognitive interventions in enhancing the learning skills of children, then the learning software can also be used as a study aid not only for children with a learning disability but for the improved performance of normal school-going children too (Law, 2017). 


\section{CONCLUSION}

This paper concludes that the use of computer-assisted intervention as remediation for learning disabilities has a lot of implications in India. The interventions provided with the help of technology can be reached to every nook and corner of the country, where the availability of a therapist is not possible thus assisting in the management of learning problems efficiently. The timely reach of interventions to the children who have a learning disability will ensure that the gap between them and their peers is not widened further. Such a fulfilling use of technology can become a boon for our country's future.

\section{REFERENCES}

(2018). Annual State education Report.

Bracy, O. L. (1999). The effects of cognitive rehabilitation therapy techniques for enhancing the cognitive/intellectual functioning of seventh and eighth grade children. International journal of cognitive technology, 19-27.

Brown, A. L. (1996). Psychological theory and the design of innovative learning environments: On procedures, principles, and systems. In L. Schauble, \& R. Glaser (Eds.), Innovations in learning: New environments for education.

Brunswick, N. M. (1999). Explicit and implicit processing of words and pseudowords by adult developmental dyslexics: a search for Wernicke's Wortschatz? Brain 122, doi: 10.1093/brain/122.10.1901, 1901-1917.

Callaghan, P. (2016). A Randomized controlled trial of an early intervention ,computer based litercy program to boost phonological skills in 4-to6-year old children. British Journal of Educational Psychology, 546-558.

Callahan, C. D. (2001). The Traumatic Brain Injury Act Amendments of 2000. Journal ofHead Trauma Rehabilitation, 210-213.

Cavanaugh, J. C. (1985). On missing links and such: interfaces between cognitive research and everyday problem solving. Human Development, 146-168.

Das, J. P. (1995). An experiment on cognitive remediation or word-reading difficulty. Journal of Learning Disabilities, 28(2), Journal of Learning Disabilities, 28(2).

Das, J. P. (2000). Cognitive education and reading disability. In A. Kozulin \& B. Y. Rand (eds.), Experience of mediated learning: An impact of Feuerstein's theory in education and psychology. Oxford: Press.

DeFelipe.J. (2006). Brain plasticity and mental processes: cajal again. Nat. Rev. Neurosci. 7,10.1038/nrn2005, 811-817.

Evans J., S. T. (1996). Rationality and reasoning. East Sussex, United Kingdom: Psychology Press.

Gathercole, S. \&. (2008). Working Memory \& Learning: A Practical Guide for Teachers. London: SAGE Publication.

Golden, G. (1982). Neurobiological correlates of learning disabilities. Ann Neurol, 409-418.

Gustafson, S. F. (2011). Effects of three interventions on the reading skills of children with reading disabilities in Grade 2. Journal of Learning Disabilities, 44(2), , 123-135.

Hayward, D. D. (2007). Evidence for innovative programs for improvement in reading: Two studies of Canadian children of First Nations. Journal of Learning Disabilities, 40, 443-457.

K.H.Pribram. (1973). The Primate Frontal Cortex Executive Of The brain. Psychophysiology of the Frontal Lobes.

Kolb, B. \&. (2011). Brain plasticity and behaviour in the developing brain. Journal of the Canadian Academy of Child and Adolescent Psychiatry=Journal de l'Academie canadienne de psychiatrie de l'enfant et de l'adolescent, 20(4), 265-276. 


\section{Computer-Assisted Cognitive Re-Training as An Intervention for Children with Specific Learning Disability: A Review}

Kujala T, K. K. (2001). Plastic neural changes and reading improvement caused by audiovisual training in reading-impaired children. Proc Natl Acad Sci U S A.98(18).

Kumar, R. C. (2014). Effectiveness of Computer Assisted Instructional Package as Remedial Teaching for Learning disabled Children. Learning Community: 5(2 and 3): August and December, 163-171.

Kuriyan, N. M. (2018). Prevalence of Learning Disability in India: A Need for Mental Health Awareness Programme. 10.4103/0253-. .

Law, M. (2017). Effect of Computer-Assisted Intervention On Early Phonological Processing Skills for Kindergarten Children In HongKong. International Journal of information and education technology. Vol7, No.12.

Macaruso, P. R. (2009). Benefits Of computer -Assisted instruction for struggling readers in middle school. European Journal of special Education Needs.

Maguire EA, et al. (2000.). Navigation-related structura lchange in the hippocampi of taxi drivers. Proc Natl Acad Sci USA 97: 4398-4403.

Malhotra, S. \&. (2010). Comparative Efficacy of Cognitive Retraining Techniques and Remedial Training in Children with Learning Disability. Delhi Psychiatry Journal. 13.

Malhotra, S. R. (2009). Efficacy of cognitive retraining techniques in children with learning disability. Delhi Psychiatry Journal, 12(1), 100-106.

Mioduser, D. T.-K. (2000). The learning value of computer-based instruction of early reading skills. Journal of Computer Assisted Learning, 16, 54-63.

Neville H, B. D. (2002). Human brain plasticity: evidence from sensory deprivation and altered language experience. Prog Brain Res 138, 177-188.

Nisha, V. K. (2013). The Efficacy of Computer Assisted Cognitive Training in the Remediation of Specific Learning Disorders. International Journal of Scientific and Research Publications (IJSRP), Volume 3, Issue 3.

Pantelidis, V. S. (1993). Virtual reality in the classroom. Educational Technology, 33(4),, 2327.

Patten, B. M. (1990). The history of memory arts. Neurology, 40, , 346-352.

Pollatsek, A. \&. (1982). Eye movement control during reading:The role of word boundaries. Journal of Experimental Psychology: Human Perception and Performance, 8, 817833.

Rogowsky, B. P. (2013). Neuroplasticity-based cognitive and linguistic skills training improves reading and writing skills in college students. Frontiers in Psychology, 4(137), 1-11.

Rozario.J., K. M. (1994). Effectiveness of Remedial education in children with reading disorders. NIMHANS Journal(12), 153-156.

Salem-Darrow M. (1996). Virtual reality's increasing potential for meeting needs of person with disabilities: what about cognitive impairments? In: $H \quad J$ Murphy (ed),Proceedings of the 3rd International Conference on Virtual Reality and Persons with Disabilities.

Schulte-Körne G1, D. W. (1998). Auditory processing and dyslexia: evidence for a specific speech processing deficit. Neuroreport.Jan 26;9(2), 337-40.

Seidman LJ, B. J. (2001). Learning disabilities and executive dysfunction in boys with attention-deficit/hyperactivity disorder. Neuropsychology.15(4), 544-556.

Shaywitz, S. (2003). Overcoming dyslexia: A new and complete science-based program for reading problems at any level.

Shaywitz, S. (2004). Reading disability and the Brain. Educational Leadership. Volume 61, 6-11.

(c) The International Journal of Indian Psychology, ISSN 2348-5396 (e)| ISSN: 2349-3429 (p) | 1047 


\section{Computer-Assisted Cognitive Re-Training as An Intervention for Children with Specific Learning Disability: A Review}

Shaywitz., S. (1998). Dyslexia. National England Journal of Medicine, 307-312.

Smythe, I. (2005). Provision and Use of Information technology with Dyslexic students in the university of Europe. EU funded project, Welsh Dyslexia society.

Standen, P.J., Brown, D.J., Cromby, J.J. (2001). The effective use of virtual environments in the education and rehabilitation of students with intellectual disabilities. Br. J. Educ. Technol. 32(3),, 289-299.

Swanson, J. R. (2011). Understanding the Effects of Stimulant Medications on Cognition in Individuals with Attention-Deficit Hyperactivity Disorder: A Decade of Progress. Neuropsychopharmacology.Jan; 36(1), 207-226.

Tallal, P. ( Sept 30, 2015). Congress hearing on the Research Excellence and Advancements for Dyslexia (READ) Act,.

Tallal, P. M. (1974). Developmental aphasia: Rate of auditory processing and selective impairment of consonant perception. Neuropsychologia 12, 83-94.

Vincent, D. ,. (1992). "Learning Difficulties and computers" Access to the Curriculum. London and Philadelphia: Jessica Kingsley Publishers.

\section{Acknowledgement}

We would like to thank Dr. Vinay Singh of orangeneurosciences.com for providing his assistance, valuable insight, and comments that greatly improved this research review paper in innumerable ways.

\section{Conflict of Interest}

The author(s) declared no conflict of interest.

How to cite this article: Bansal G. \& Singh A. P. (2021). Computer-Assisted Cognitive ReTraining as An Intervention for Children with Specific Learning Disability: A Review. International Journal of Indian Psychology, 9(4), 1038-1048. DIP:18.01.099.20210904, DOI:10.25215/0904.099 